\title{
Adaptive Control of Delayed Teleoperation Systems with Parameter Convergence
}

\author{
Yuling Li 1 , Yixin Yin, and Sen Zhang $(1)$ \\ School of Automation and Electrical Engineering, University of Science and Technology Beijing, Beijing 100083, China \\ Correspondence should be addressed to Yuling Li; lyl8ustb@gmail.com
}

Received 7 November 2017; Accepted 6 March 2018; Published 5 April 2018

Academic Editor: Calogero Orlando

Copyright (C) 2018 Yuling Li et al. This is an open access article distributed under the Creative Commons Attribution License, which permits unrestricted use, distribution, and reproduction in any medium, provided the original work is properly cited.

\begin{abstract}
It is well known that parameter convergence in adaptive control can bring about an improvement of system performance, including accurate online identification, exponential tracking, and robust adaptation without parameter drift. However, strong persistentexcitation (PE) or sufficient-excitement (SE) conditions should be satisfied to guarantee parameter convergence in the classical adaptive control. This paper proposes a novel adaptive control to guarantee parameter convergence without PE and SE conditions for nonlinear teleoperation systems with dynamic uncertainties and time-varying communication delays. The stability criterion of the closed-loop teleoperation system is given in terms of linear matrix inequalities. The effectiveness of this approach is illustrated by simulation studies, where both master and slave are assumed to be two-link manipulators with full nonlinear system dynamics.
\end{abstract}

\section{Introduction}

Bilateral teleoperation systems are one of the most wellknown time-delay systems which allow a human operator to extend his/her intelligence and manipulation skills to the remote environments. A typical teleoperation system is composed of five parts: human operator, master, communication channel, slave, and task environment. The master is directly handled by a human operator to manipulate the slave in the task environment, and the signals (position, velocity, or interaction force) from the slave are sent back to the master to improve the performance. Recent years have witnessed considerable advances in the control studies of teleoperation systems, owing to their broad engineering applications in telesurgery, space exploration, nuclear operation, undersea exploration, and so forth.

It is well known that the information between the master and slave robots is transmitted via a communication network, and long-distance data transmission generally causes communication time delays. The existence of such communication time delays may affect the stability and the control performance of teleoperation systems. In order to solve this problem, a variety of control schemes have been proposed in the literature. The breakthrough work on bilateral teleoperation problem was achieved in [1], in which the concepts from network theory, passivity, and scattering theory were used to analyze the stability of the controlled teleoperation systems [2], and then the master-slave synchronization and stability analysis of teleoperation systems with various kinds of time delays, such as constant delays [1], time-varying delays [3-8], or stochastic delays [9-11], have been hot topics in the study of teleoperation systems.

Another typical concern for teleoperation systems is the dynamic uncertainties. For model-based controllers, the system parameters are assumed to be explicitly known. However, it is unrealistic to accurately get the system model in practical applications. Therefore, one of the main solutions to reduce the influence of uncertainty on the performance of a teleoperation system is to design an adaptive controller. For nonlinear teleoperation systems, the design of adaptive controllers mainly uses a basic fact: the master-slave robots are linearly parameterized [12]. In [13], an adaptive controller for teleoperators with time delays, which ensures synchronization of positions and velocities of the master and slave manipulators and does not rely on the use of the ubiquitous scattering transformation, was proposed. Based on [13], two versions of adaptive controllers for nonlinear bilateral teleoperators were proposed in [5]. The authors in [14] proposed a novel adaptive control framework for 
nonlinear teleoperation systems with dynamic and kinematic uncertainties and time-varying time delays. Unfortunately, one of the drawbacks of adaptive controllers proposed in $[5,13,14]$ is that the parameter estimates are not guaranteed to converge to the true parameters. It is well known that the convergence of the parameters to their true values can improve system performance with accurate online identification, exponential tracking, and robust adaptation without parameter drift. Unfortunately, these features are not guaranteed unless a condition of persistent excitation $(\mathrm{PE})$ is satisfied [15]. However, the PE condition is very stringent and often infeasible in practical control systems [16]. Hence, some improved methods which relax the condition of PE should be proposed.

Motivated by the above concerns, in this paper, a new adaptive controller is designed for teleoperation systems with time-varying delays, and the convergence of parameters to their true values is achieved, which then gives rise to an improvement of system performance. A new prediction error is designed to guarantee the parameter convergence, and the condition of $\mathrm{PE}$ is not required; thus, the proposed control scheme is more practical in real applications.

The arrangement of this paper is as follows. In Section 2, the system modeling and some preliminaries are given. In Section 3, the adaptive control with parameter convergence is given and its stability is analyzed in Section 4. A simple teleoperation system composed of two robots with two degrees of freedom is given as an example to show the effectiveness of the proposed method in Section 5. Finally, the summary and conclusion of this paper are given in Section 6.

Notations. Throughout this paper, the superscript $T$ stands for matrix transposition. $\mathbb{R}^{n}$ denotes the $n$-dimensional Euclidean space with vector norm $\|\cdot\| \cdot \mathbb{R}^{n \times m}$ is the set of all $n \times m$ real matrices. $*$ represents a block matrix which is readily referred by symmetry. $\lambda_{\min }(M)$ and $\lambda_{\max }(M)$ denote the maximum and the minimum eigenvalue of matrix $M=$ $M^{T} \in \mathbb{R}^{n \times n}$, respectively. For any function $f:[0, \infty) \rightarrow \mathbb{R}^{n}$, the $\mathscr{L}_{\infty}$-norm is defined as $\|f\|_{\infty}:=\sup _{t \geqslant 0}|f(t)|$, and the square of the $\mathscr{L}_{2}$-norm is defined as $\|f\|_{2}^{2}:=\int_{0}^{\infty}|f(t)|^{2} d t$. The $\mathscr{L}_{\infty}$ and $\mathscr{L}_{2}$ spaces are defined as the sets $\{f:[0, \infty) \rightarrow$ $\left.\mathbb{R}^{n},\|f\|_{\infty}<\infty\right\}$ and $\left\{f:[0, \infty) \rightarrow \mathbb{R}^{n},\|f\|_{2}<\infty\right\}$, respectively.

\section{Problem Formulation and Preliminaries}

Consider teleoperation systems described as follows:

$$
\begin{array}{r}
M_{m}\left(q_{m}\right) \ddot{q}_{m}+C_{m}\left(q_{m}, \dot{q}_{m}\right) \dot{q}_{m}+G_{m}\left(q_{m}\right)=F_{m}+\tau_{m} \\
M_{s}\left(q_{s}\right) \ddot{q}_{s}+C_{s}\left(q_{s}, \dot{q}_{s}\right) \dot{q}_{s}+G_{s}\left(q_{s}\right)=F_{s}+\tau_{s},
\end{array}
$$

where $q_{i}, \dot{q}_{i}, \ddot{q}_{i} \in \mathbb{R}^{n}$ are the vectors of the joint position, velocity, and acceleration with $i=m$ or $s$ representing the master or the slave robot manipulator, respectively. Similarly, $M_{i}$ represents the mass matrix, and $C_{i}\left(q_{i}, \dot{q}_{i}\right)$ embodies the Coriolis and centrifugal effects. $\tau_{i}$ is the control force, and finally $F_{m}, F_{s}$ are the external forces applied to the manipulator end-effectors. Each robot in (1) satisfies the structural properties of robotic systems, that is, the following properties $[2,12]$ :

(P1) The inertia matrix $M_{i}\left(q_{i}\right)$ is a symmetric positivedefinite function and is lower and upper bounded; that is, $0<\rho_{i}^{m} I \leqslant M_{i}\left(q_{i}\right) \leqslant \rho_{i}^{M} I<\infty$, where $\rho_{i}^{m}, \rho_{i}^{M}$ are positive scalars.

(P2) The matrix $\dot{M}_{i}\left(q_{i}\right)-2 C_{i}\left(q_{i}, \dot{q}_{i}\right)$ is skew symmetric.

(P3) For all $q_{i}, x, y \in \mathbb{R}^{n \times 1}$, there exists a positive scalar $c_{i}$ such that $\left\|C_{i}\left(q_{i}, x\right) y\right\| \leqslant c_{i}\|x\|\|y\|$.

(P4) The equations of motion of $n$-link robot can be linearly parameterized as

$$
\begin{aligned}
& M_{i}\left(q_{i}\right) \ddot{q}_{i}+C_{i}\left(q_{i}, \dot{q}_{i}\right) \dot{q}_{i}+G_{i}\left(q_{i}\right)=Y_{i o}\left(q_{i}, \dot{q}_{i}, \ddot{q}_{i}\right) \theta \\
& \quad \triangleq y_{i},
\end{aligned}
$$

where $Y_{i o}\left(q_{i}, \dot{q}_{i}, \ddot{q}_{i}\right) \triangleq Y_{i o} \in \mathbb{R}^{n \times n_{i}}$ is a matrix of known functions called regressor and $\theta_{i} \in \mathbb{R}^{n_{i}}$ is a vector of unknown parameters.

In this paper, the data is transmitted from the master to the slave and from the slave to the master over delayed communication with variable delays. The communication delays are assumed to have certain bounds, which is precisely stated in Assumption 1.

Assumption 1. For each $i=m, s$, the variable communication time delay $T_{i}(t)$ has a known upper bound $h_{i}(t)$; that is, $0 \leqslant$ $T_{i}(t) \leqslant h_{i}$. Additionally, the time derivative $\dot{T}_{i}$ is bounded.

\section{Adaptive Control Design}

Suppose the positions of the master and the slave are available for measurement and are transmitted through the delayed network communication. Let $e_{i} \in \mathbb{R}^{n}$ denote the position errors by

$$
\begin{gathered}
e_{m} \triangleq q_{m}-q_{s}\left(t-T_{s}(t)\right) \\
e_{s} \triangleq q_{s}-q_{m}\left(t-T_{m}(t)\right) .
\end{gathered}
$$

We define the following auxiliary variables:

$$
\begin{gathered}
\eta_{m} \triangleq \dot{q}_{m}+\lambda_{m} e_{m} \\
\eta_{s} \triangleq \dot{q}_{s}+\lambda_{s} e_{s},
\end{gathered}
$$

where $\lambda_{m}, \lambda_{s}$ are positive constants. Using property (P4), letting $Y_{i} \theta_{i}=Y_{i}\left(q_{i}, \dot{q}_{i}, e_{i}, \dot{e}_{i}\right) \theta_{i}=M_{i}\left(q_{i}\right) \lambda_{i} \dot{e}_{i}+C_{i}\left(q_{i}, \dot{q}_{i}\right) \lambda_{i} e_{i}-$ $G_{i}\left(q_{i}\right), i \in\{m, s\}$, the following control laws for the master and the slave are proposed:

$$
\begin{gathered}
\tau_{m}=-Y_{m} \hat{\theta}_{m}-K_{m} \eta_{m} \\
\tau_{s}=-Y_{s} \hat{\theta}_{s}-K_{s} \eta_{s},
\end{gathered}
$$

where $\widehat{\theta}_{i}$ is the estimate of $\theta_{i}, K_{i}>0$.

Substituting the control law (6) into the teleoperation dynamics (1), we obtain the following dynamics for $t>0$ :

$$
\begin{gathered}
M_{m}\left(q_{m}\right) \dot{\eta}_{m}+C_{m}\left(q_{m}, \dot{q}_{m}\right) \eta_{m}+K_{m} \eta_{m}=Y_{m} \tilde{\theta}_{m}+F_{m} \\
M_{s}\left(q_{s}\right) \dot{\eta}_{s i}+C_{s}\left(q_{s}, \dot{q}_{s}\right) \eta_{s}+K_{s} \eta_{s}=Y_{s} \tilde{\theta}_{s}+F_{s},
\end{gathered}
$$

where $\widetilde{\theta}_{i} \triangleq \theta_{i}-\widehat{\theta}_{i}$. 
A straightforward choice of the adaptive law $\dot{\hat{\theta}}_{i}=\Gamma_{i} Y_{i}^{T} \eta_{i}$ was first proposed by Slotine and Li [17] and has been widely used in adaptive control of teleoperation systems [2, 5, 13]. However, it is pointed out that this adaptive law cannot guarantee accurate estimations of parameters. In order to achieve convergence of parameters to their true values, the estimation error $\widetilde{\theta}_{i}$ should be introduced into the control design. However, the value of $\widetilde{\theta}_{i}$ is not obtainable since the true value of $\theta_{i}$ is not available, and thus the prediction error $e_{i o}=Y_{i o} \widetilde{\theta}_{i}$ or its filtered counterpart $e_{i w}=\alpha \int_{0}^{t} e^{-\delta(t-v)} e_{i o}(v) d v$ is used to improve the tracking performance. However, the use of $e_{i o}$ or $e_{i w}$ still needs the PE condition to make the system exponentially stable. In the following, we introduce an auxiliary variable $z_{i}$ such that $z_{i}=P_{i} \widetilde{\theta}_{i}$, where $P_{i}$ is a designed lower bounded positive-definite matrix, to adaptive control of the teleoperation system. Thus, the following adaptive laws are proposed for the master and the slave:

$$
\begin{aligned}
\dot{\hat{\theta}}_{i} & =\Gamma_{i}\left(Y_{i}^{T} \eta_{i}+\left(\xi_{i}+\delta_{i}\right) z_{i}\right) \\
\dot{z}_{i} & =-\mu_{i} z_{i}+Y_{i o}^{T} e_{i o}-P_{i} \dot{\hat{\theta}}_{i}, \\
z_{i}(0) & =0 \\
\dot{P}_{i} & =-\mu_{i} P_{i}+Y_{i o}^{T} Y_{i o}, \\
P_{i}(0) & =P_{i 0}>0 \\
\mu_{i} & =\mu_{i 0}\left(1-\kappa_{i 0}\left\|P_{i}^{-1}\right\|\right),
\end{aligned}
$$

where

$$
\begin{aligned}
& e_{m o} \triangleq y_{m}-Y_{m o} \widehat{\theta}_{m}=Y_{m o} \tilde{\theta}_{m} \\
& e_{s o} \triangleq y_{s}-Y_{s o} \widehat{\theta}_{s}=Y_{s o} \widetilde{\theta}_{s},
\end{aligned}
$$

where $\kappa_{i 0}$ and $\mu_{i 0}$ are two positive constants specifying the lower bound of the norm of $P_{i}$ and the maximum forgetting rate [17]; $\Gamma_{m} \in \mathbb{R}^{n_{m} \times n_{m}}$ and $\Gamma_{s} \in \mathbb{R}^{n_{s} \times n_{s}}$ are two constant positive-definite matrices. From (10) and (11), one can show that, $\forall t \geqslant 0, \mu_{i} \geqslant 0$, and $P_{i} \geqslant \kappa_{i 0} I$.

The coefficient $\xi_{i}$ is given by

$$
\xi_{i}=\alpha_{i} \frac{\left\|Y_{i}^{T} \eta_{i}\right\|}{\kappa_{i 0}},
$$

where $\alpha_{i}>0$ is a constant.

Remark 2. By (12), it is easy to see that the prediction error $e_{i o}$ is related to the regressor $Y_{i}$, which requires the information of joint acceleration. To avoid this, the adaptive law ((8)-(11)) with filtered torques and filtered regressor $Y_{i w}$ could be used. The filtered prediction errors of estimated parameters are defined as

$$
\begin{gathered}
e_{m w} \triangleq y_{m w}-Y_{m w} \widehat{\theta}_{m}=Y_{m w} \widetilde{\theta}_{m} \\
e_{s w} \triangleq y_{s w}-Y_{s w} \widehat{\theta}_{s}=Y_{s w} \widetilde{\theta}_{s},
\end{gathered}
$$

where $y_{i}$ is the filtered forces $\tau_{i}+F_{i}$, that is,

$$
y_{i w}=\alpha \int_{0}^{t} e^{-\alpha(t-\delta)} y_{i} d \delta,
$$

and can be calculated without acceleration terms $M_{i}\left(q_{i}\right) \ddot{q}_{i}$ by convolving both sides of (2) by a filter $W(s)=\alpha /(s+\alpha)$ [18].

\section{Stability Analysis}

Denote $x_{m}=\left[\eta_{m}^{T}, \tilde{\theta}_{m}\right]^{T}, x_{s}=\left[\eta_{s}^{T}, \tilde{\theta}_{s}\right]^{T}$, and $x=\left[x_{m}^{T}, x_{s}^{T}\right]^{T}$ and define the new state $x_{t}(s) \triangleq x(t+s), s \in[-h, 0]$ which take values in $C\left([-h, 0] ; \mathbb{R}^{n+m+s}\right), h=\max \left\{h_{m}, h_{s}\right\}$.

The following theorem summarizes the main result of this paper.

Theorem 3. Consider the bilateral teleoperation system (1) controlled by (6) together with the updating law ((8)-(11)) under the communication channel satisfying Assumption 1, if there exist positive-definite matrices $R_{m}, R_{s}$ such that the following linear matrix inequality (LMI) holds, respectively:

$$
\Pi=\left[\begin{array}{cccc}
\Pi_{1} & 0 & 0 & -I \\
* & \Pi_{2} & -I & 0 \\
* & * & -\frac{R_{m}}{h_{m}} & 0 \\
* & * & * & -\frac{R_{s}}{h_{s}}
\end{array}\right]<0,
$$

with

$$
\begin{aligned}
& \Pi_{1}=-\frac{1}{\lambda_{m}} I+h_{m} R_{m}, \\
& \Pi_{2}=-\frac{1}{\lambda_{s}} I+h_{s} R_{s},
\end{aligned}
$$

and then when the considered teleoperation system is in free motion, that is, $F_{m}=F_{s}=0$, all the signals are bounded and the position errors, velocities, and estimation errors asymptotically converge to zero; that is, $\lim _{t \rightarrow \infty} q_{m}-q_{s}=\lim _{t \rightarrow \infty} \dot{q}_{m}=$ $\lim _{t \rightarrow \infty} \dot{q}_{s}=\lim _{t \rightarrow \infty} \tilde{\theta}_{m}=\lim _{t \rightarrow \infty} \tilde{\theta}_{s}=0$. Moreover, the estimation errors converge to a specified domain within a given time.

Proof. Define the following function:

$$
V_{i}(x, t)=\frac{1}{2} \eta_{i}^{T} M_{i}\left(q_{i}\right) \eta_{i}+\frac{1}{2} \widetilde{\theta}_{i}^{T} \Gamma_{i}^{-1} \widetilde{\theta}_{i}
$$

It is obvious that $V_{i}$ is positive-definite and radially unbounded with regard to $\eta_{i}$ and $\widetilde{\theta}_{i}$. Using property (P2), the derivative of $V_{i}$ along the trajectory of system (7) is

$$
\begin{aligned}
\dot{V}_{i}(x, t) & =-\eta_{i}^{T} K_{i} \eta_{i}-\widetilde{\theta}_{i}^{T}\left(\xi_{i} P_{i}+\delta_{i} P_{i}\right) \tilde{\theta}_{i} \\
& \leqslant-\eta_{i}^{T} K_{i} \eta_{i}-\delta_{i} \kappa_{i 0} \widetilde{\theta}_{i}^{T} \widetilde{\theta}_{i}
\end{aligned}
$$

when $F_{m} \equiv 0, F_{s} \equiv 0$. Since $V_{i}(x, t)>0, \dot{V}_{i}(x, t)<0$, we conclude that $\eta_{i}, \widetilde{\theta}_{i} \in \mathscr{L}_{\infty} \cap \mathscr{L}_{2}$. By the closed-loop dynamics (7) 
and properties (P1)-(P4), we have that $\dot{\eta}_{i} \in \mathscr{L}_{\infty}$. Thus, by Barbalat's Lemma, one has that $\lim _{t \rightarrow \infty} \eta_{i}(t)=0$. Now, we give the following Lyapunov functional:

$$
V=V_{1}+V_{2}+V_{3}
$$

with

$$
\begin{aligned}
& V_{1}=\frac{1}{k_{m} \lambda_{m}} V_{m}(x, t)+\frac{1}{k_{s} \lambda_{s}} V_{s}(x, t) \\
& V_{2}=\left(q_{m}-q_{s}\right)^{T}\left(q_{m}-q_{s}\right) \\
& V_{3}=\sum_{i=m, s} \int_{-h_{i}}^{0} \int_{t+\theta}^{t} \dot{q}_{i}^{T}(s) R_{i} \dot{q}_{i}(s) d s d \theta,
\end{aligned}
$$

where $k_{i}=\lambda_{\min }\left\{K_{i}\right\}$.

When the external forces $F_{m} \equiv F_{s} \equiv 0$, by (4), the derivative of $V_{1}$ along with the trajectory of system (7) is given by

$$
\begin{aligned}
& \dot{V}_{1}(x, t)=\frac{1}{k_{m} \lambda_{m}} \dot{V}_{m}(x, t)+\frac{1}{k_{s} \lambda_{s}} \dot{V}_{s}(x, t) \\
& \quad \leqslant-\sum_{i=m, s}\left(\frac{\dot{q}_{i}^{T} \dot{q}_{i}}{\lambda_{i}}+2 e_{i}^{T} \dot{q}_{i}+\lambda_{i} e_{i}^{T} e_{i}+\frac{\delta_{i} \kappa_{i 0}}{k_{i}} \widetilde{\theta}_{i}^{T} \widetilde{\theta}_{i}\right) .
\end{aligned}
$$

It is noted that the position error $e \triangleq q_{m}-q_{s}$ can be expressed as

$$
e=e_{m}-L_{s}=-e_{s}+L_{m}
$$

where $L_{m}=\int_{t-T_{m}(t)}^{t} \dot{q}_{m}(s) d s$ and $L_{s}=\int_{t-T_{s}(t)}^{t} \dot{q}_{s}(s) d s$, and hence the time derivative of $V_{2}$ along with the trajectory of system (7) is given by

$$
\dot{V}_{2}=2\left(e_{m}-L_{s}\right)^{T} \dot{q}_{m}+2\left(e_{s}-L_{m}\right)^{T} \dot{q}_{s} .
$$

Calculating the time derivative of $V_{3}$, one has that

$$
\begin{aligned}
\dot{V}_{3} & =\sum_{i=m, s} h_{i} \dot{q}_{i}^{T} R_{i} \dot{q}_{i}-\int_{t-h_{i}}^{t} \dot{q}_{i}^{T}(s) R_{i} \dot{q}_{i}(s) d s \\
& \leqslant \sum_{i=m, s} h_{i} \dot{q}_{i}^{T} R_{i} \dot{q}_{i}-\int_{t-T_{i}(t)}^{t} \dot{q}_{i}^{T}(s) R_{i} \dot{q}_{i}(s) d s \\
& \leqslant \sum_{i=m, s} h_{i} \dot{q}_{i}^{T} R_{i} \dot{q}_{i}-\frac{1}{h_{i}} L_{i} R_{i} L_{i}
\end{aligned}
$$

by Jensen's inequality.

Thus, we have

$$
\dot{V}=\sum_{i=1,2,3} \dot{V}_{i} \leqslant-\xi \Pi \xi-\sum_{i=m, s}\left(\frac{\delta_{i} \kappa_{i 0}}{k_{i} \lambda_{i}}\left|\widetilde{\theta}_{i}\right|^{2}+\lambda_{i} e_{i}^{T} e_{i}\right),
$$

where $\xi=\operatorname{col}\left\{\dot{q}_{m}, \dot{q}_{s}, L_{m}, L_{s}\right\}$ and $\Pi$ is given in (17).

By (17), we have that $\dot{V}<0$ and $V>0$. Furthermore, by (21) and (27), one has that $e_{i} \in \mathscr{L}_{2} \cap \mathscr{L}_{\infty}, \widetilde{\theta}_{i} \in \mathscr{L}_{2} \cap \mathscr{L}_{\infty}$. Thus, by (3), one has that $\dot{e}_{m}, \dot{e}_{s} \in \mathscr{L}_{\infty}$ if the time derivatives of the communication delays $\dot{T}_{i}$ are bounded. Now, invoking Barbalat's Lemma, we conclude that $\lim _{t \rightarrow \infty} e_{i}(t)=0$. Thus, it is followed by $\lim _{t \rightarrow \infty} \dot{q}_{i}(t)=0$ since $\eta_{i} \rightarrow 0$ as $t \rightarrow \infty$. Since $e=e_{m}-L_{s}=-e_{s}+L_{m}$, we further conclude that $e \rightarrow 0$ as $t \rightarrow \infty$.

Now, we show that the parameter estimation error $\widetilde{\theta}_{i}$ approaches zero as $t \rightarrow \infty$. Note that the parameter adaption law (8) can be represented as

$$
\dot{\tilde{\theta}}_{i}=-\Gamma_{i}\left(Y_{i}^{T} \eta_{i}+\left(\xi_{i}+\delta_{i}\right) P_{i} \widetilde{\theta}_{i}\right) \in \mathscr{L}_{\infty}
$$

Similarly, the conclusion that $\lim _{t \rightarrow \infty} \widetilde{\theta}_{i}(t)=0$ is guaranteed using Barbalat's Lemma.

To illustrate the transient performance of the teleoperators, we start from the convergence of estimation errors $\widetilde{\theta}_{i}$. Obviously, the Lyapunov candidate function for $\widetilde{\theta}_{i}$ is $\sum_{i=m, s}\left(1 / k_{i} \lambda_{i}\right) \widetilde{\theta}_{i}^{T} \Gamma_{i}^{-1} \widetilde{\theta}_{i}$, which we denoted as $V_{\theta}(t)$. The time derivative of $V_{\theta}$ is given by

$$
\begin{aligned}
& \dot{V}_{\theta}(t)=\sum_{i=m, s} \frac{1}{k_{i} \lambda_{i}} \widetilde{\theta}_{i}^{T} \Gamma_{i}^{-1} \dot{\tilde{\theta}}_{i} \\
& \leqslant \sum_{i=m, s} \frac{1}{k_{i} \lambda_{i}}\left(-\widetilde{\theta}_{i}^{T} Y_{i}^{T} \eta_{i}-\widetilde{\theta}_{i}^{T} \alpha_{i}\left\|Y_{i}^{T} \eta_{i}\right\| \widetilde{\theta}_{i}-\delta_{i} \kappa_{i 0} \widetilde{\theta}_{i}^{T} \widetilde{\theta}_{i}\right) \\
& \leqslant \sum_{i=m, s} \frac{1}{k_{i} \lambda_{i}}\left(\left(1-\alpha_{i}\left\|\widetilde{\theta}_{i}\right\|\right)\left\|Y_{i}^{T} \eta_{i}\right\|\left\|\widetilde{\theta}_{i}\right\|-\delta_{i} \kappa_{i 0}\left\|\widetilde{\theta}_{i}\right\|^{2}\right) .
\end{aligned}
$$

Thus, if $\left\|\theta_{i}\right\| \geqslant 1 / \alpha_{i}$ for both $i=m, s$, we have $\dot{V}_{\theta}(t) \leqslant$ $-\sum_{i=m, s}\left(\delta_{i} \kappa_{i 0} / k_{i} \lambda_{i}\right)\left\|\widetilde{\theta}_{i}\right\|^{2} \leqslant-\delta\|\widetilde{\theta}\|^{2}$, where $\delta=\min \left\{\delta_{m} \kappa_{m 0} /\right.$ $\left.k_{m} \lambda_{m}, \delta_{s} \kappa_{s 0} / k_{s} \lambda_{s}\right\}$. This implies that $\dot{V}_{\theta}$ is always negative when $\|\theta\| \geqslant \sqrt{2} / \alpha$ with $\alpha=\min \left\{\alpha_{m}, \alpha_{s}\right\}$. So, the parameter error $\widetilde{\theta}(t)=\operatorname{col}\left\{\widetilde{\theta}_{m}, \widetilde{\theta}_{s}\right\}$ will converge to a sphere $\Omega_{\theta}=$ $\left\{\tilde{\theta}:\|\tilde{\theta}\| \leqslant \sqrt{2 \lambda_{M}^{\theta} \nu_{M} / \lambda_{m}^{\theta} \nu_{m}} / \alpha\right\}$, where $\lambda_{m}^{\theta}=\min \left\{\lambda_{\min }\left(\Gamma_{m}^{-1}\right)\right.$, $\left.\lambda_{\text {min }}\left(\Gamma_{s}^{-1}\right)\right\}, \lambda_{M}^{\theta}=\max \left\{\lambda_{\max }\left(\Gamma_{m}^{-1}\right), \lambda_{\max }\left(\Gamma_{s}^{-1}\right)\right\}, \nu_{m}=\min \{1 /$ $\left.k_{m} \lambda_{m}, 1 / k_{m} \lambda_{m}\right\}$, and $\nu_{M}=\max \left\{1 / k_{m} \lambda_{m}, 1 / k_{m} \lambda_{m}\right\}$ within a given time.

Remark 4. Compared to the existing works $[5,13,19]$, the proposed control scheme guarantees the convergence of parameters to their true values, while the condition of $\mathrm{PE}$ is not required. This is accomplished by the boundedness of the matrix $P_{i}$ in the new-defined prediction error $z_{i}$.

\section{Simulations}

In this section, the simulation results are shown to verify the effectiveness of the main result. Consider a 2-DOF teleoperation system (1) with the following parameters:

$$
\begin{gathered}
M_{i}\left(q_{i}\right)=\left[\begin{array}{lll}
M_{i_{11}}\left(q_{i}\right) & M_{i_{12}}\left(q_{i}\right) \\
M_{i_{21}}\left(q_{i}\right) & M_{i_{22}}\left(q_{i}\right)
\end{array}\right], \\
C_{i}\left(q_{i}, \dot{q}_{i}\right)=\left[\begin{array}{ll}
C_{i_{11}}\left(q_{i}, \dot{q}_{i}\right) & C_{i_{12}}\left(q_{i}, \dot{q}_{i}\right) \\
C_{i_{21}}\left(q_{i}, \dot{q}_{i}\right) & C_{i_{22}}\left(q_{i}, \dot{q}_{i}\right)
\end{array}\right], \\
G_{i}\left(q_{i}\right)=\left[\begin{array}{l}
G_{i_{1}} \\
G_{i_{2}}
\end{array}\right],
\end{gathered}
$$




$$
\begin{gathered}
F_{m}=J_{m}^{T} f_{h}, \\
F_{s}=J_{s}^{T} f_{e},
\end{gathered}
$$

for $i=m, s$, respectively, and

$$
\begin{aligned}
& M_{i_{11}}\left(q_{i}\right) \\
& \quad=l_{i_{2}}^{2} m_{i_{2}}+l_{i_{1}}^{2}\left(m_{i_{1}}+m_{i_{2}}\right)+2 l_{i_{1}} l_{i_{2}} m_{i_{2}} \cos \left(q_{i_{2}}\right), \\
& M_{i_{22}}\left(q_{i}\right)=l_{i_{2}}^{2} m_{i_{2}}, \\
& M_{i_{12}}\left(q_{i}\right)=M_{i_{21}}\left(q_{i}\right)=l_{i_{2}}^{2} m_{i_{2}}+l_{i_{1}} l_{i_{2}} m_{i_{2}} \cos \left(q_{i_{2}}\right), \\
& C_{i_{11}}\left(q_{i}, \dot{q}_{i}\right)=-l_{i_{1}} l_{i_{2}} m_{i_{2}} \sin \left(q_{2_{i}}\right) \dot{q}_{i_{2}}, \\
& C_{i_{12}}\left(q_{i}, \dot{q}_{i}\right)=-l_{i_{1}} l_{i_{2}} m_{i_{2}} \sin \left(q_{2_{i}}\right)\left(\dot{q}_{1_{i}}+\dot{q}_{2_{i}}\right) \\
& C_{i_{21}}\left(q_{i}, \dot{q}_{i}\right)=l_{i_{1}} l_{i_{2}} m_{i_{2}} \sin \left(q_{2_{i}}\right) \dot{q}_{1_{i}}, \\
& C_{i_{22}}\left(q_{i}, \dot{q}_{i}\right)=0, \\
& G_{i_{1}}\left(q_{i}\right) \\
& \quad=\frac{1}{l_{i_{2}}} g l_{i_{2}}^{2} m_{i_{2}} \cos \left(q_{1_{i}}+q_{2_{i}}\right) \\
& \quad+\frac{1}{l_{i_{1}}}\left(l_{i_{2}}^{2} m_{i_{2}}+l_{i_{1}}^{2}\left(m_{i_{1}}+m_{i_{2}}\right)-l_{i_{2}}^{2} m_{i_{2}}\right) \cos \left(q_{1_{i}}\right) \\
& G_{i_{2}}\left(q_{i}\right)=\frac{1}{l_{i_{2}}} g l_{i_{2}}^{2} m_{i_{2}} \cos \left(q_{1_{i}}+q_{2_{i}}\right) .
\end{aligned}
$$

The masses of the manipulators are chosen as $m_{m_{1}}=1.5 \mathrm{~kg}$, $m_{m_{2}}=0.75 \mathrm{~kg}, m_{s_{1}}=2.5 \mathrm{~kg}$, and $m_{s_{2}}=1.5 \mathrm{~kg}$, and the lengths of links for the master and the slave robots are $l_{m_{1}}=l_{s_{1}}=0.5 \mathrm{~m}$ and $l_{m_{2}}=l_{s_{2}}=0.3 \mathrm{~m}$. The Jacobians of the master and slave robots are given by $J_{i}\left(q_{i}\right)=$ $\left[-l_{i_{1}} \sin \left(q_{i_{1}}\right)-l_{i_{2}} \sin \left(q_{i_{1}}+q_{i_{2}}\right)-l_{i_{2}} \sin \left(q_{i_{1}}+q_{i_{2}}\right)\right]$.

$\left[\begin{array}{ll}l_{i_{1}} \cos \left(q_{i_{1}}\right)+l_{i_{2}} \cos \left(q_{i_{1}}+q_{i_{2}}\right) & l_{i_{2}} \cos \left(q_{i_{1}}+q_{i_{2}}\right)\end{array}\right]$.

The following parameterization is proposed for both manipulators with $i=m, s$, respectively:

$$
\begin{aligned}
& Y_{i}\left(q_{i}, \dot{q}_{i}, \ddot{q}_{i}\right) \\
& =\left[\begin{array}{ccccc}
\ddot{q}_{i_{1}} & Y_{12} & \ddot{q}_{i_{2}} & g \cos \left(q_{i_{1}}+q_{i_{2}}\right) & g \cos \left(q_{i_{1}}\right) \\
0 & Y_{22} & \ddot{q}_{i_{1}}+\ddot{q}_{i_{2}} & g \cos \left(q_{i_{1}}+q_{i_{2}}\right) & 0
\end{array}\right], \\
& Y_{12} \\
& =2 \cos \left(q_{i_{2}}\right) \ddot{q}_{i_{1}}+\cos \left(q_{i_{2}}\right) \ddot{q}_{i_{2}}-2 \sin \left(q_{i_{2}}\right) \dot{q}_{i_{1}} \dot{q}_{i_{2}} \\
& -\sin \left(q_{i_{2}}\right) \dot{q}_{i_{2}}^{2}, \\
& Y_{22}=\cos \left(q_{i_{2}}\right) \ddot{q}_{i_{1}}+\sin \left(q_{i_{2}}\right) \dot{q}_{i_{1}}^{2}, \\
& \theta_{i}=\operatorname{col}\left\{\theta_{i_{1}}, \theta_{i_{2}}, \theta_{i_{3}}, \theta_{i_{4}}, \theta_{i_{5}}\right\} \text {, }
\end{aligned}
$$

where $\theta_{i_{1}}=l_{i_{2}}^{2} m_{i_{2}}+l_{i_{1}}^{2}\left(m_{i_{1}}+m_{i_{2}}\right), \theta_{i_{1}}=l_{i_{2}}^{2} m_{i_{2}}+l_{i_{1}}^{2}\left(m_{i_{1}}+m_{i_{2}}\right)$, $\theta_{i_{3}}=l_{i_{2}}^{2} m_{i_{2}}, \theta_{i_{4}}=l_{i_{2}} m_{i_{2}}$, and $\theta_{i_{5}}=l_{i_{1}}\left(m_{i_{1}}+m_{i_{2}}\right)$. We assume

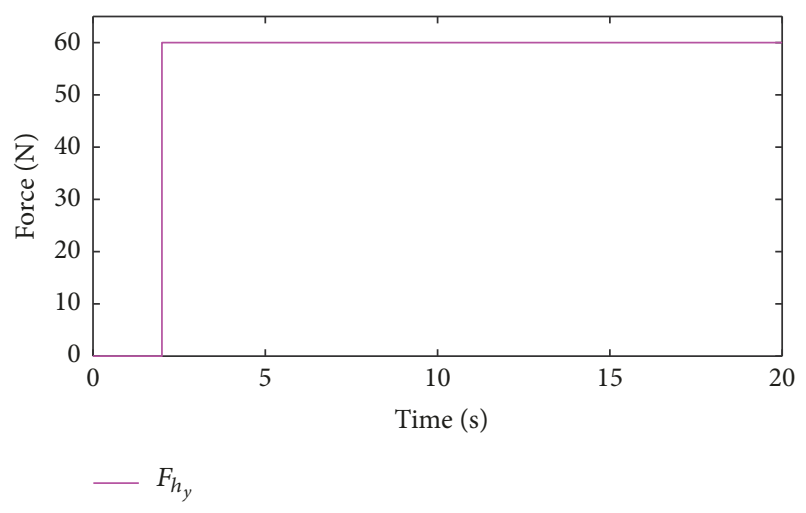

FIGURE 1: The rectangle human input force.

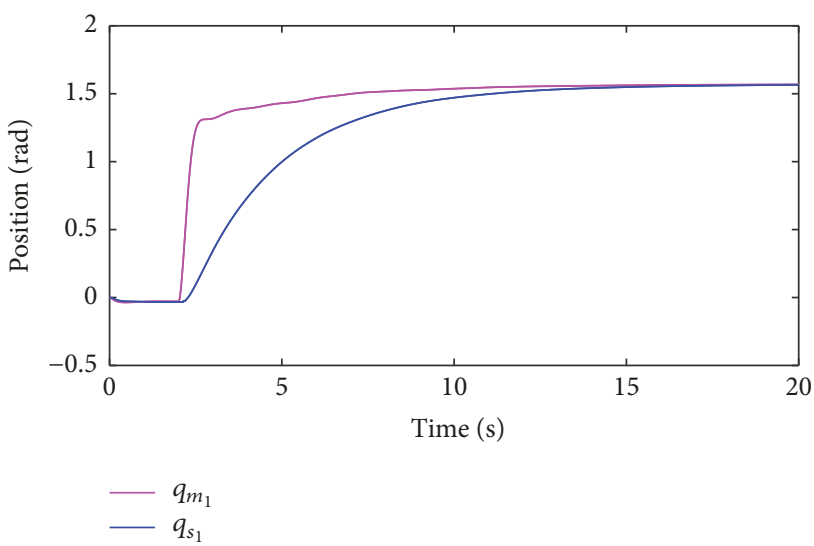

FIGURE 2: The master and slave robots position: link 1 .

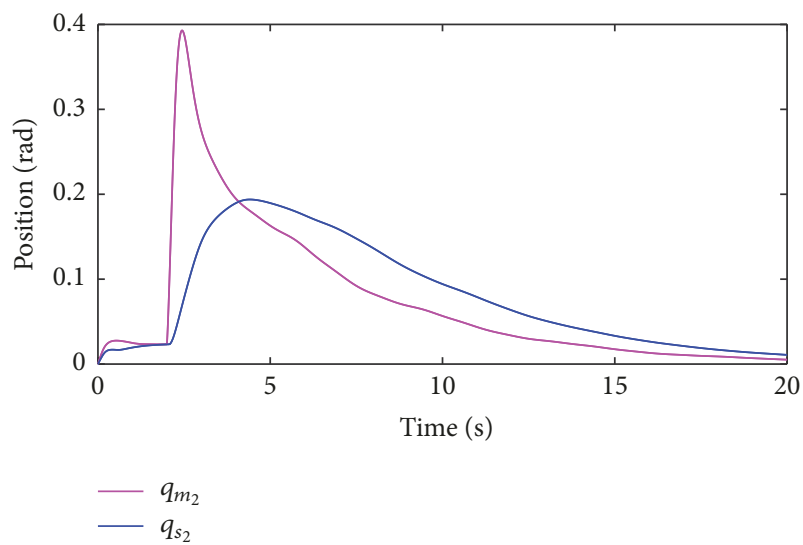

FIGURE 3: The master and slave robots position: link 2 .

that the operator hand force at the $Y$-direction is generated by a step signal depicted in Figure 1, while at the $X$-direction, there is no external force; then, we have $F_{h}=[0,1]^{T} F_{h_{y}}$. The slave is in free motion in this simulation. By applying the designed controller (6), (8), (9), (10), and (11) with $K_{m}=$ $K_{s}=5 I, \lambda_{m}=\lambda_{s}=0.5$, we obtain the simulation results as shown in Figures 2 and 3. It can be seen that, under the proposed controller, the presence of parametric uncertainties 


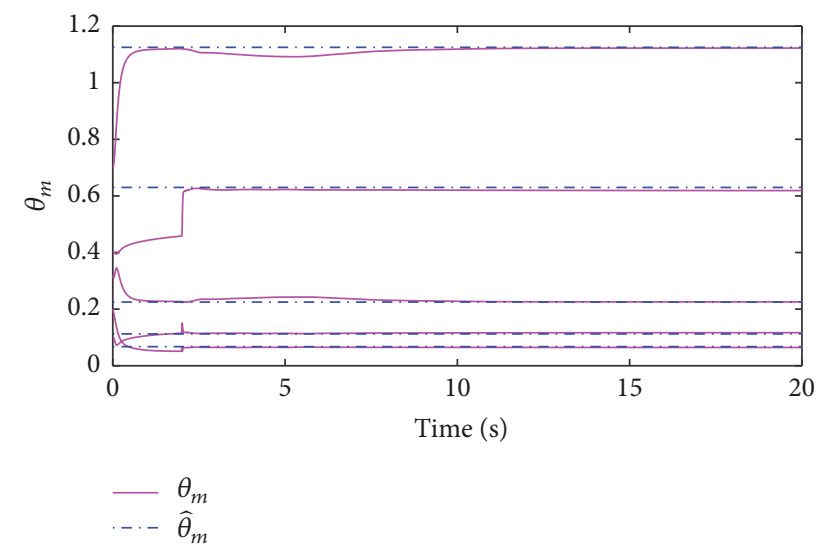

FIgURE 4: The dynamic parameter $\theta_{m}$ and the parameter estimate $\widehat{\theta}_{m}$.

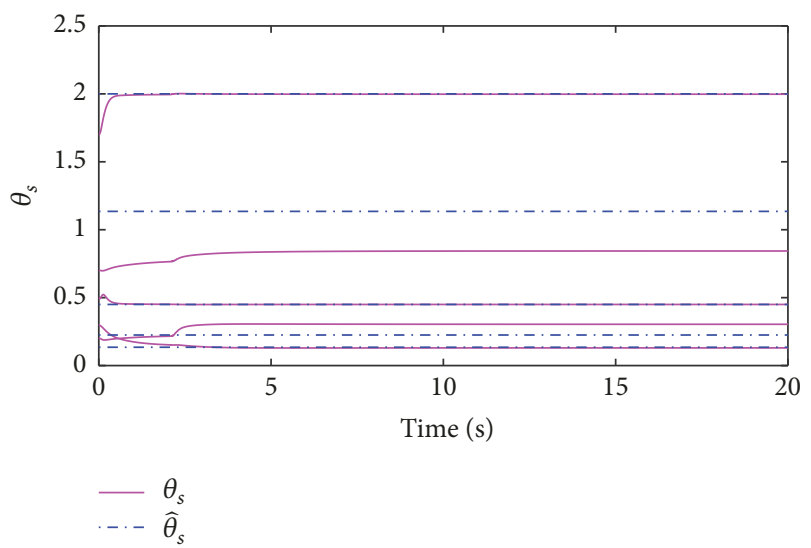

FIGURE 5: The dynamic parameter $\theta_{s}$ and the parameter estimate $\widehat{\theta}_{s}$.

does not violate the stability of the bilateral teleoperation. The master and the slave achieve synchronization around the time $t=10 \mathrm{~s}$. Furthermore, the estimated dynamic parameters are shown in Figures 4 and 5, respectively. By Figure 4, it is easy to find that the estimate $\widehat{\theta}_{m}$ converges to its real value $\theta_{m}$ after $t=2 \mathrm{~s}$, at which time the external human force starts to be exerted to the master manipulator. Similarly, Figure 5 reveals the convergence of the slave's dynamic parameters to their true values.

\section{Conclusion}

In this paper, a novel adaptive control framework that addressed dynamic uncertainties and time-varying delays for nonlinear teleoperation systems was proposed. Contrary to the existing works which guarantee the boundedness of the parameter estimation errors, this paper achieves convergence of parameters to their true values, which then gives rise to an improvement of system performance. By designing a new prediction error, the condition of $\mathrm{PE}$ is relaxed in this paper. The controller performance is verified via simulations. Further studies on parameter-converging adaptive control of teleoperation systems with a configuration of single master and multiple slaves are underway and the results will be reported in the near future.

\section{Conflicts of Interest}

The authors declare that there are no conflicts of interest regarding the publication of this paper.

\section{Acknowledgments}

This work was jointly supported by the National Natural Science Foundation of China (nos. 61333002 and 61773053), the Fundamental Research Funds for the Central Universities of USTB (nos. FRF-TP-16-024A1, FPR-BD-16-005A, and FRF-GF-17-A4), the Beijing Key Discipline Development Program (no. XK100080537), and the Beijing Natural Science Foundation (no. 4182039).

\section{References}

[1] R. J. Anderson and M. W. Spong, "Bilateral control of teleoperators with time delay," Institute of Electrical and Electronics Engineers Transactions on Automatic Control, vol. 34, no. 5, pp. 494-501, 1989.

[2] E. Nuño, L. Basañez, and R. Ortega, "Passivity-based control for bilateral teleoperation: a tutorial," Automatica, vol. 47, no. 3, pp. 485-495, 2011.

[3] C. C. Hua and X. P. Liu, "Delay-dependent stability criteria of teleoperation systems with asymmetric time-varying delays," IEEE Transactions on Robotics, vol. 26, no. 5, pp. 925-932, 2010.

[4] Y. Li, R. Johansson, K. Liu, and Y. Yin, "Guaranteed cost control design for delayed teleoperation systems," Journal of The Franklin Institute, vol. 352, no. 11, pp. 5085-5105, 2015.

[5] I. Sarras, E. Nuño, and L. Basañez, "An adaptive controller for nonlinear teleoperators with variable time-delays," Journal of The Franklin Institute, vol. 351, no. 10, pp. 4817-4837, 2014.

[6] Y. Yang, C. Hua, and X. Guan, "Synchronization control for bilateral teleoperation system with prescribed performance under asymmetric time delay," Nonlinear Dynamics, vol. 81, no. 1-2, pp. 481-493, 2015.

[7] D.-H. Zhai and Y. Xia, "Adaptive finite-time control for nonlinear teleoperation systems with asymmetric time-varying delays," International Journal of Robust and Nonlinear Control, vol. 26, no. 12, pp. 2586-2607, 2016.

[8] D.-H. Zhai and Y. Xia, "Adaptive Control for Teleoperation System with Varying Time Delays and Input Saturation Constraints," IEEE Transactions on Industrial Electronics, vol. 63, no. 11, pp. 6921-6929, 2016.

[9] Y. Kang, Z. Li, X. Cao, and D. Zhai, "Robust control of motion/force for robotic manipulators with random time delays," IEEE Transactions on Control Systems Technology, vol. 21, no. 5, pp. 1708-1718, 2013.

[10] Z. Li, Y. Xia, D. Wang, D.-H. Zhai, C.-Y. Su, and X. Zhao, "Neural Network-Based Control of Networked Trilateral Teleoperation with Geometrically Unknown Constraints," IEEE Transactions on Cybernetics, vol. 46, no. 5, pp. 1051-1064, 2016.

[11] Z. Li and Y. Xia, "Adaptive neural network control of bilateral teleoperation with unsymmetrical stochastic delays and unmodeled dynamics," International Journal of Robust and Nonlinear Control, vol. 24, no. 11, pp. 1628-1652, 2014. 
[12] W. Mark Spong, S. Hutchinson, and V. Mathukumalli, Robot modeling and control, vol. volume 3, Wiley, New York, 2006.

[13] E. Nuño, R. Ortega, and L. Basañez, "An adaptive controller for nonlinear teleoperators," Automatica, vol. 46, no. 1, pp. 155-159, 2010.

[14] Y. Li, Y. Yin, and D. Zhang, "Adaptive Task-Space Synchronization Control of Bilateral Teleoperation Systems With Uncertain Parameters and Communication Delays," IEEE Access, vol. 6, pp. 5740-5748, 2018.

[15] Y. Pan and H. Yu, "Composite learning from adaptive dynamic surface control," Institute of Electrical and Electronics Engineers Transactions on Automatic Control, vol. 61, no. 9, pp. 2603-2609, 2016.

[16] Y. Pan, T. Sun, L. Pan, and H. Yu, "Robustness analysis of composite adaptive robot control," in Proceedings of the 28th Chinese Control and Decision Conference, CCDC 2016, pp. 510515, China, May 2016.

[17] E. Jean-Jacques Slotine and W. Li, Applied nonlinear control. prentice-Hall, NJ, Englewood Cliffs, 1991.

[18] B.-Y. Kim and H.-S. Ahn, "A design of bilateral teleoperation systems using composite adaptive controller," Control Engineering Practice, vol. 21, no. 12, pp. 1641-1652, 2013.

[19] N. Chopra, M. W. Spong, and R. Lozano, "Synchronization of bilateral teleoperators with time delay," Automatica, vol. 44, no. 8, pp. 2142-2148, 2008. 


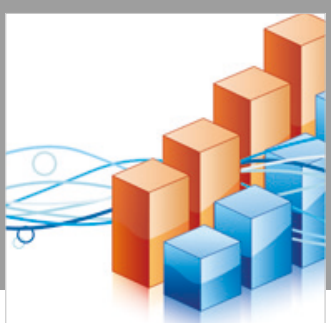

Advances in

Operations Research

\section{-n-m}
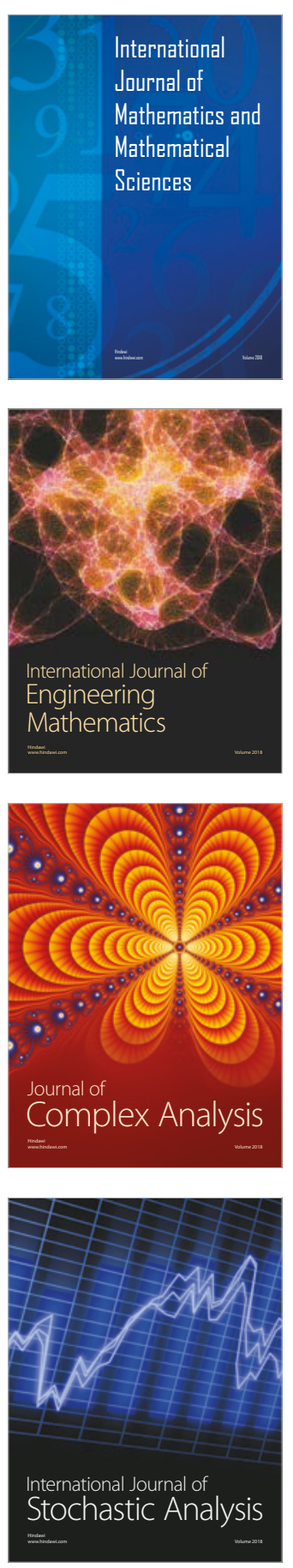
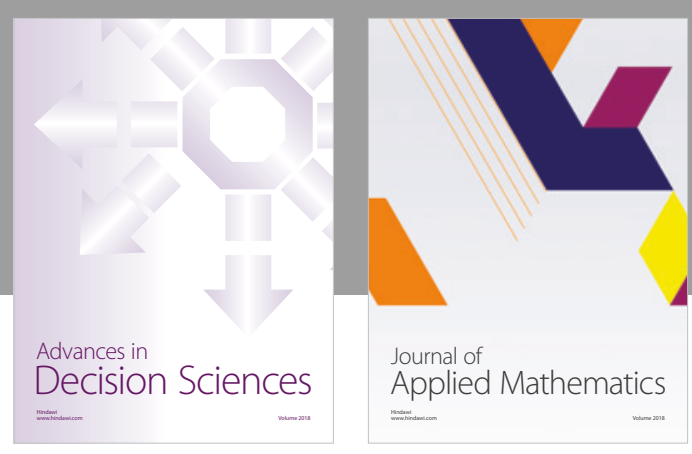

Journal of

Applied Mathematics
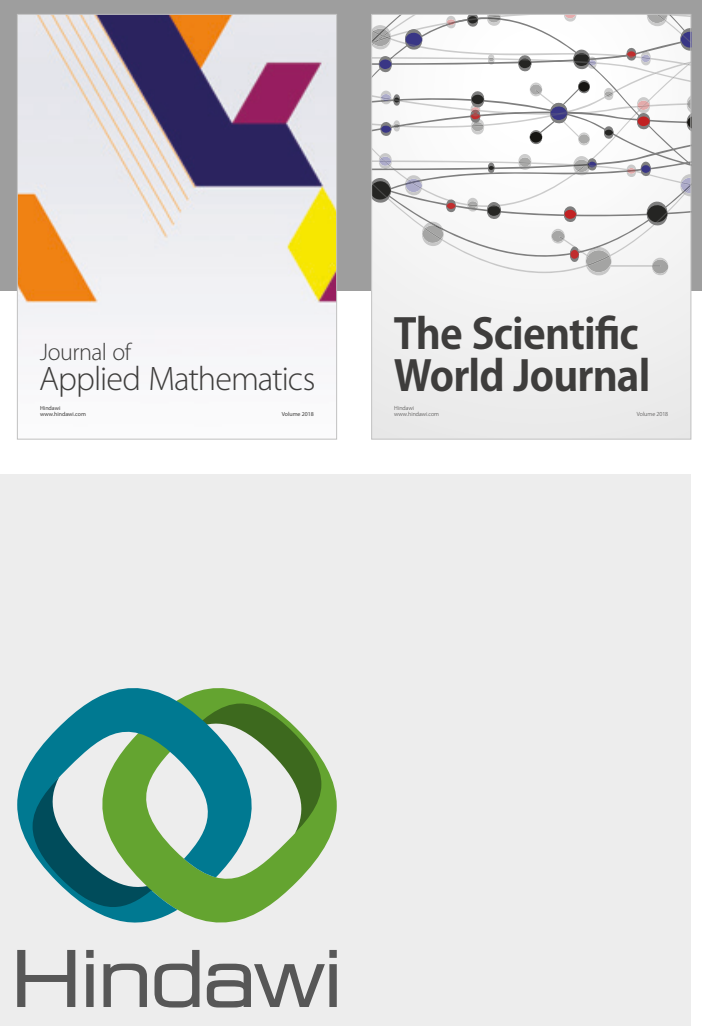

Submit your manuscripts at

www.hindawi.com

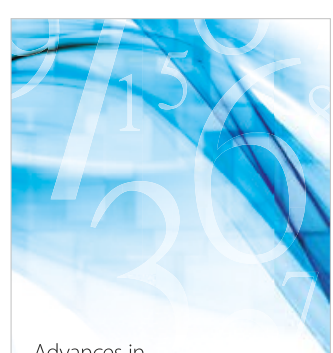

Advances in
Numerical Analysis
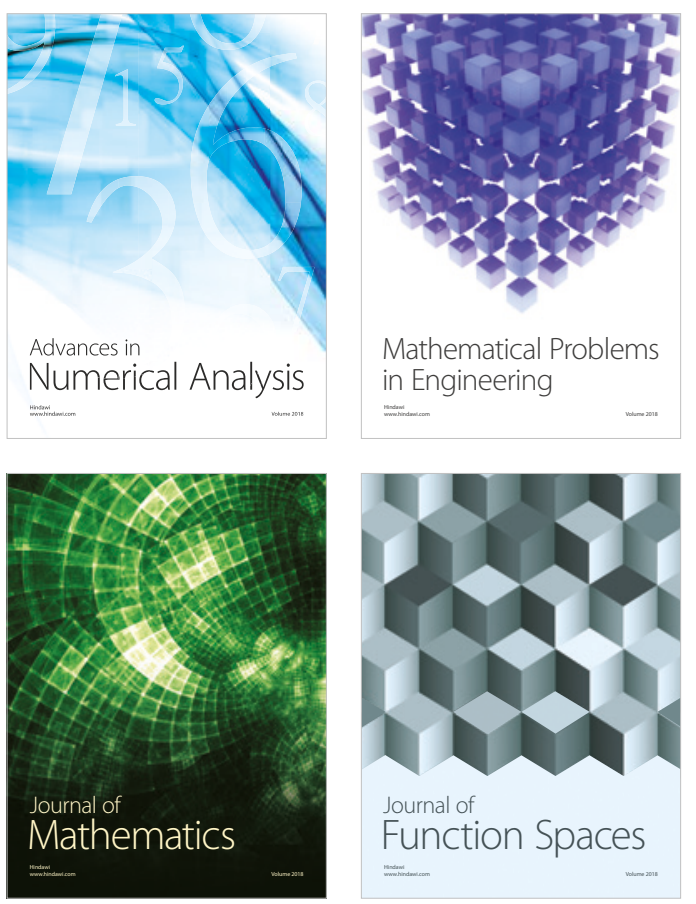

Mathematical Problems in Engineering

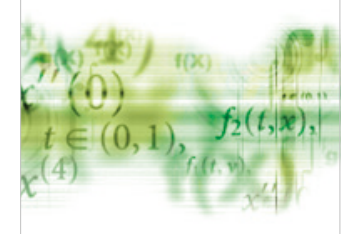

International Journal of

Differential Equations

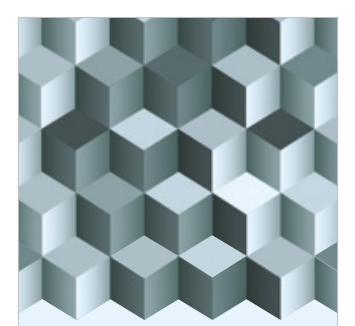

Journal of

Function Spaces

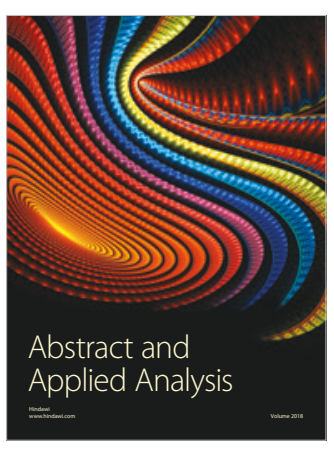

The Scientific

World Journal

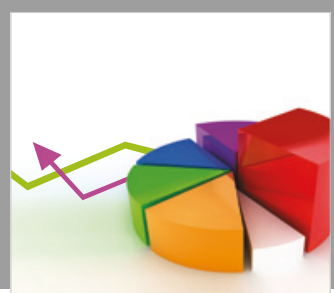

Journal of

Probability and Statistics
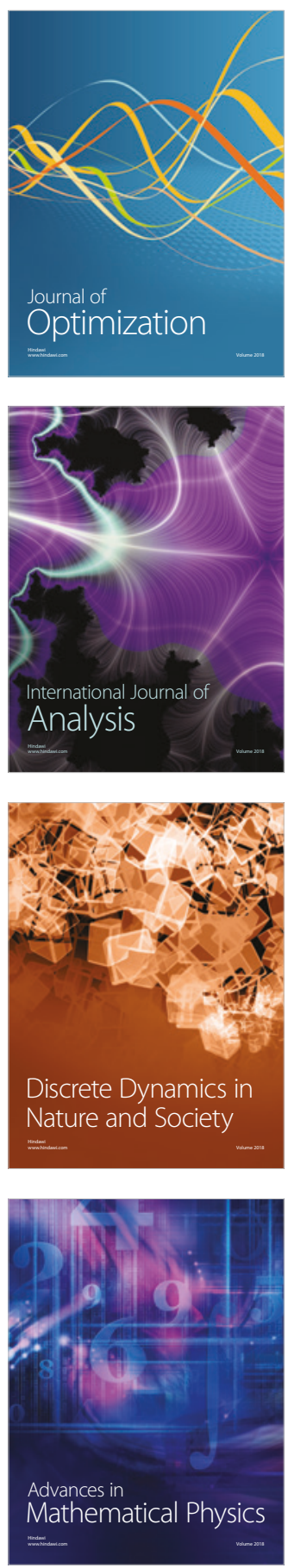